\title{
Deviation from bulk behaviour in the cold crystallization kinetics of ultrathin films of poly(3-hydroxybutyrate), PHB
}

\author{
S Napolitano and M Wübbenhorst \\ Katholieke Universiteit Leuven \\ Laboratory of Acoustics and Thermal Physics \\ Department of Physics and Astronomy \\ Celestijnenlaan 200D, \\ B-3001 Leuven, Belgium \\ Email: michael.wubbenhorst@fys.kuleuven.be
}

KEYWORDS: cold crystallization, ultrathin polymer films, poly(3-hydroxybutyrate)

PACS: 36.20.-r, 65.40.-b, 64.70.Pf, 65.60.+a, 65.80.+n, 68.60.Dv, 73.61.Ph, 77.22.Gm, 77.84.Jd, 78.20.Ci, 82.35.Lr, 87.15.Nn.

\begin{abstract}
The cold crystallization kinetics of ultrathin films of poly(3-hydroxybutyrate) (PHB) have been investigated by dielectric spectroscopy. Upon reduction of the film thickness, a lowering of the Avrami exponent accompanied by an increase of the crystallization time was observed. The experimental results are analyzed in terms of reduction of the total number of nuclei involved in the crystallization process.
\end{abstract}

\section{Introduction}

The tremendous changes in the molecular dynamics and in the material properties occurring in confined geometry when the dimension of the samples is reduced to a few tens of nanometres, have being fascinating the scientific community for more than ten years ${ }^{1}$. Several simulations ${ }^{2-5}$ and experimental techniques ${ }^{6-9}$ were applied to study the transition from the supercooled liquid state to the glassy state, characterised by the glass transition temperature $\left(T_{\mathrm{g}}\right)$, in nanometres-thick specimens polymer layers with the specific aim to detect size-related deviations from bulk behaviour. Though most of the work has proven the existence of strong confinement effects on $T_{\mathrm{g}}$, the partially contradictive observations have hampered the development of a consistent physical picture of the effects in confinements so far. 
More consistent results come from the experimental and theoretical studies of the crystallization in ultrathin films ${ }^{10-18}$. A variety of different experimental approaches took to results being qualitatively to the same trend: in case of strong interactions between the polymer and the substrate onto which the film was prepared, a huge reduction of the crystallization rate is observed, i.e. an increase of the crystallization time and the cold crystallization temperature (defined as the temperature at which the inverse of the crystallization time equals the heating rate), and reduction of the linear crystals' growth rate and the Avrami exponent.

Recently, it was shown that dielectric spectroscopy, DS, can detect the changes in the amorphous volume fraction during crystallization even for polymer samples with nanometre dimensions ${ }^{18}$. Contrarily to the other techniques previously employed to study the slowing-down of the crystallization kinetics in ultrathin polymer films, DS can provide a detailed analysis of the segmental mobility of the analyzed samples. It was then demonstrated that an increase of the crystallization time (by more than one order of magnitude) is not imputable to a corresponding reduction of the segmental mobility on the time and lengthscale of the glass $\operatorname{transition}^{18}$, i.e. the crystallization time can increase even if the dynamic glass transition temperature of the sample remains constant.

A possible explanation for the reduction of the crystallization kinetics is the presence of a region with a lower mobility in the proximity to the substrate ${ }^{18}$. Such a region would play a key role on the dynamics of the whole polymer film, when the dimension of the sample is comparable with the lengthscale of the interfacial interaction. For these samples a pronounced slow-down of the crystallisation kinetics is expected. Another cause for changes in the kinetics is the reduction of the number of active nuclei taking part to the conversion of the amorphous phase into crystals. Due to a finite value of the nuclei density, the probability $\eta(h)$ to find a nucleus in a thin layer decreases with the reduction of the thickness and tends to zero for the thinnest films. This last explanation is supported by several theoretical model $\mathrm{s}^{20-22}$ and fits the experimental results obtained in this work

In this paper we argue that dielectric spectroscopy can be applied to extract kinetics parameters of the cold crystallization of ultrathin polymer films. Combining this information with the analysis of changes in the dielectric spectra at the reduction of the thickness ${ }^{18}$, it is possible to add new details to the comprehension of the origin of the tremendous deviations from bulk behaviour in ultrathin polymer films.

\section{Experimental}

Poly(3-hydroxyburate) in powder by Sigma $\left(\mathrm{M}_{\mathrm{w}}=170 \mathrm{k}\right)$, was melted for 5 minutes $15 \mathrm{~K}$ above the melting point $\left(\mathrm{T}_{\mathrm{m}}{ }^{\mathrm{DSC}}=443 \mathrm{~K}\right)$ between two brass circular electrodes. Bulk amorphous samples were produced by quenching the molten layer between two cold plates held at $268 \mathrm{~K}$, below the glass transition temperature $\left(\mathrm{T}_{\mathrm{g}}{ }^{\mathrm{DSC}}=275 \mathrm{~K}\right)$. The final thickness of the sample was given by the diameter of the glass fibres $(\varnothing=50 \mu \mathrm{m})$ used to separate the brass electrodes in order to avoid shortcuts.

Ultrathin films of PHB were prepared by spincoating very dilute solutions (down to $0,2 \% \mathrm{w} / \mathrm{w}$ ) of the polymer that was dissolved in chloroform and filtered by a Teflon membrane filter (Millipore). Aluminium electrodes were evaporated in an ultra-high vacuum chamber on cleaned glass slides and were used as 
substrate for the samples. Spincoating was performed at room temperature, thus well above $\mathrm{T}_{\mathrm{g}}$. After an annealing procedure of 2 hours at $318 \mathrm{~K}$, the samples were slowly cooled down to room temperature and a second aluminium layer, providing the upper electrode, was evaporated onto the polymer surface.

During the two metal evaporations the temperature on the sample did not exceed room temperature. Film thicknesses were evaluated from the electrical capacity $C^{\prime}$ of the sample ${ }^{23}$ according to the relation $\mathrm{h}=\varepsilon^{\prime} \varepsilon_{0} \mathrm{~S} / \mathrm{C}^{\prime}$ (where $\varepsilon^{\prime}$ is the dielectric constant of a bulk sample, $\varepsilon_{0}$ is the permittivity of the vacuum and $\mathrm{S}$ the area of the region at the overlapping or the two electrodes), valid in the approximation of parallel electrodes with 'infinite' lateral dimensions.

Dielectric spectra were recorded $(10 \mathrm{mHz}-10 \mathrm{MHz})$ with a high resolution dielectric analyzer (ALPHA-A from Novocontrol Technologies) immediately after amorphization in order to avoid nucleation processes due to storage of the sample above the glass transition temperature.

Spectra of the bulk quenched samples were characterized by a relatively intense peak, $\alpha$, attributed to the structural relaxation, and a weaker peak, $\beta$, due to the relaxation of the ester group within the chain bone ${ }^{24}$, in the same temperature and frequency range as reported for amorphous samples of this polymer ${ }^{25}$.

The $\alpha$-relaxation was not detected in the ultrathin samples obtained according the preparation procedure described above, indicating the semicrystalline nature of the films after the annealing procedure. Amorphous samples were obtained by melting and rapidly quenching the

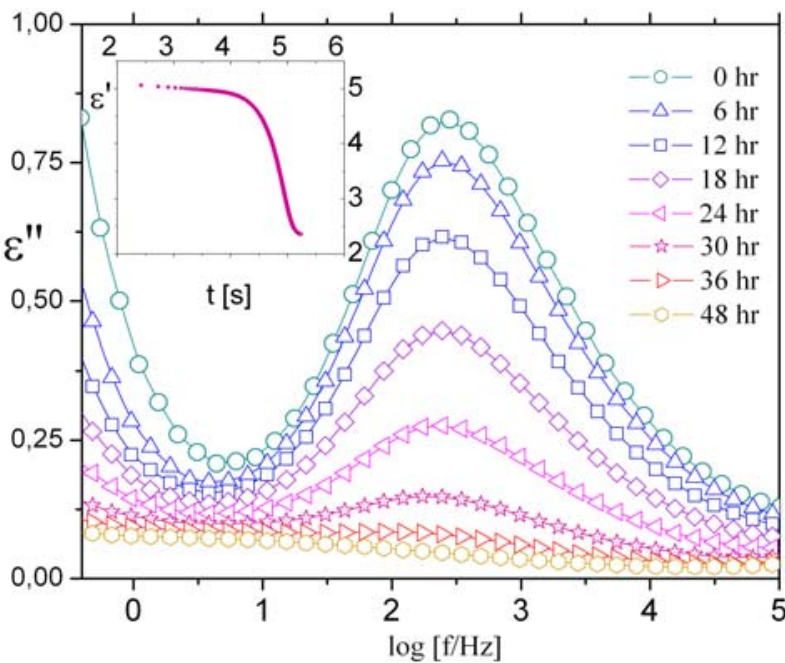

Fig 1. Dielectric spectra of a $26 \mathrm{~nm}$ thick sample during an isothermal crystallization at $291 \mathrm{~K}$. The continuous lines are guides for the eye. In the inset, the time evolution of the dielectric constant at $10 \mathrm{~Hz}$ for the analyzed samples. ultrathin layers following the procedure reported for bulk samples.

Spectra of the complex dielectric permittivity, $\varepsilon^{*}(\omega)=\varepsilon^{\prime}(\omega)-i \varepsilon^{\prime *}(\omega)$, were acquired under a continuous nitrogen flow, in isothermal conditions, with a temperature stability better than $0.1{ }^{\circ} \mathrm{C}$. Isothermal crystallization kinetics were monitored in real time by choosing the appropriate low frequency limit $(1.2 \mathrm{~Hz}$ for bulk samples and $0.49 \mathrm{~Hz}$ for the thinnest film) in order to ensure the validity of the condition $\mathrm{t}_{\mathrm{ss}}<<\mathrm{t}_{1 / 2}{ }^{\mathrm{DS}}$, where $t_{S S}$ is the time necessary to record a single spectrum, and $t_{1 / 2}{ }^{D S}$ is the mean time of the crystallization process assigned via dielectric spectroscopy ${ }^{18}$.

\section{Results and discussion}

\subsection{Changes to the dielectric spectra during crystallization kinetics}


Figure 1 shows the effect of isothermal crystallization at $291 \mathrm{~K}$ on the structural relaxation of a $26 \mathrm{~nm}$ thick sample, in terms of the reduction of the intensity of the peak and drop in the real part of the complex dielectric permittivity, in the inset. For PHB, it was proven that the changes in the spectra during the conversion of the amorphous phase are insensitive to the sample thickness, thus the following description is valid for both bulk and ultrathin films down to $26 \mathrm{~nm}$. The only difference is the time scale of the phenomena investigated ${ }^{18}$.

Within the first 3 hours changes on the spectra are not discernable; this regime corresponds to the induction time during which nucleation processes take to the formation of ordered domains. It was pointed out ${ }^{19}$ that during this waiting time pre-ordering could take place and DS resulted a more sensitive tool compared to diffraction techniques, like SAXS and WAXS.

As already reported for other polymers ${ }^{26-30}$, after the induction time the intensity of the structural relaxation decreases due to a reduction of the density of mobile dipoles. During the primary crystallization the chains rearrange in ordered structures; the crystalline regions of the sample do not contribute any longer to the dielectric response, as the molecules involved become immobile. This corresponds to a drop in the dielectric strength $\Delta \varepsilon$, and consequently to a reduction of $\varepsilon "{ }_{\text {max }}$. In the final stages of the crystallization process a second peak, connected with the relaxation of the amorphous fraction constrained within the growing lamellae was detected ${ }^{18}$.

Contrarily to other, less flexible polyesters like $\mathrm{PET}^{27,31}$ and $\mathrm{PEN}^{28}$, the mean relaxation time of the structural process appears to be insensitive to the crystalline content of sample. However, a similar trend was observed for another polymer system with flexible chains, PLLA ${ }^{32,33}$. Furthermore it was

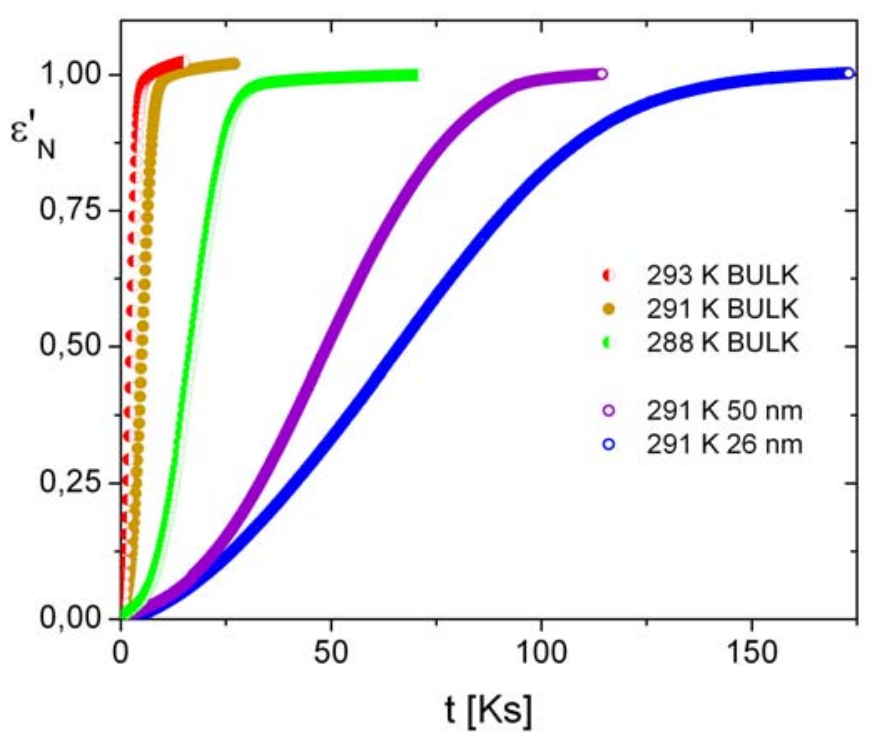

Figure 2. Time evolution of the normalized dielectric constant at $10 \mathrm{~Hz}$ for the analyzed samples. The half crystallization time $\mathrm{t}_{1 / 2}{ }^{\mathrm{DS}}$ is given by the mid point of the step in the curve. observed that for PHB, the relaxation time of the structural process does not show a dependence on the thickness of the sample ${ }^{18}$. It is thus possible to analyze the changes in the dielectric constant $\varepsilon^{\prime}(\mathrm{t})$ during the crystallization time and relate them to the changes in amorphous volume fraction via a relation $^{34,35}$ like

$$
\varepsilon_{N}^{\prime}(t)=\frac{\varepsilon(0)-\varepsilon(t)}{\varepsilon(0)-\varepsilon(\infty)}=\alpha \cdot X_{C}(t)
$$

where $\varepsilon^{\prime}{ }_{N}$ is the normalized dielectric constant, $\varepsilon^{\prime}(0)$ and $\varepsilon^{\prime}(\infty)$ are respectively the value of the dielectric constant at the beginning and at the end of the process, $X_{C}$ is the crystallinity as determined by diffraction techniques and $\alpha$ is a constant $(\alpha \leq 1)$ taking into account reduction of the dielectric signal not directly correlated to the decrease 
of the amorphous fraction. For PHB and its copolymers with poly(3-hydroxyvalerate), $\mathrm{PHV}^{24}, \alpha \approx 1$ was reported. A linear relationship between $\varepsilon^{\prime}{ }_{N}(\mathrm{t})$ and $\mathrm{X}_{\mathrm{C}}(\mathrm{t})$ is a good approximation for a likely more complex physical scenario. A semicrystalline material is a multiphase system, and thus higher-orders corrections should be taken into account to compensate interface and local field effects. These deviations should be nonnegligible in the case of non flexible polymer chains sensing the relaxation of both the constraint and the mobile amorphous phase. As for PHB $\partial \tau_{\alpha} / \partial \mathrm{t}$ is zero within the experimental errors, during the crystallization time the portion of the chains giving rise to the dielectric signal relaxes always in the same the environment. Hence, Eq (1) is valid.

Fig. 2 shows the values of $\varepsilon_{N}(T)$ at different thicknesses and temperatures. An increase of by more than one order of magnitude in $\mathrm{t}_{1 / 2}{ }^{\mathrm{DS}}$ is observed when the thickness of the sample is reduced to $26 \mathrm{~nm}$. The result agrees with similar deviations from bulk behaviour observed by different investigation techniques in other polymer systems ${ }^{10,14,15}$. Being the temperatures investigated in the regime of cold crystallization, for this polymer both the temperature and the reduction of the thickness act qualitatively on the same way: colder or thinner samples show a higher crystallization time $\mathrm{t}_{1 / 2}{ }^{\mathrm{DS}}$.

\subsection{Analysis of the isothermal crystallization kinetics}

In order to analyse the isothermal crystallization kinetics a standard approach is given by the Avrami equation $^{36}$ :

$$
1-\varphi_{C}=\exp \left(-K t^{n}\right)
$$

where $\varphi_{\mathrm{C}}$ is the crystalline volume fraction, $K$ is a constant, given by the temperature and the geometry of the sample, $n$ is the Avrami exponent; the value of this last parameter is finally correlated to the dimensionality

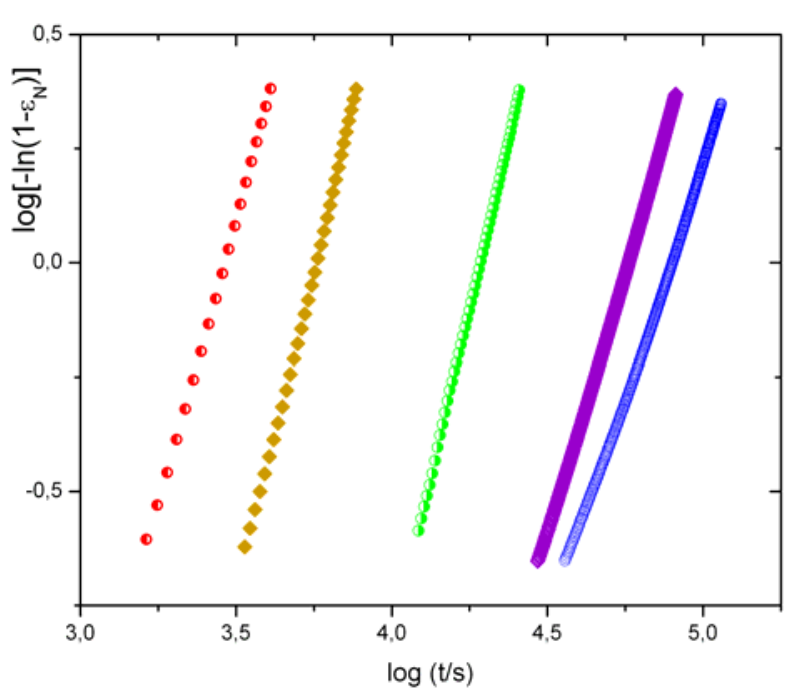

Fig 3. Avrami plot for the analyzed samples. For clarity only the data relative to a normalized dielectric constant between 0.2 and 0.9 are shown. The same symbols as in Fig.2 have been used of the process. Combining the equations (1) and (2), modified Avrami equations can be used to analyze directly data obtained by dielectric measurements ${ }^{35,37,38}$ :

$$
\begin{gathered}
\varepsilon_{N}^{\prime}(t)=1-\exp \left(-K t^{n}\right) \\
\varepsilon_{N}^{\prime}(t)=1-\exp \left[-\left(\frac{t}{\tau_{c r y}}\right)^{\beta}\right]
\end{gathered}
$$

By plotting the curves obtained via eq. (3) in an Avrami plot, i.e. $\log \left[-\ln \left(1-\varepsilon_{\mathrm{N}}\right)\right]$ vs. $\log (t)$, see Figure 3 it was possible to obtain the Avrami parameters $\mathrm{n}$ and $\mathrm{K}$ respectively as slope of the and intercept on the y-axis of the obtained straight line. Eq. (4) is a Kohlrausch-Williams-Watts equation where, $\tau_{\text {cry }}$ is the characteristic time of 
the process, and $\beta$ a constant. By comparing Eq (3) and Eq (4) we obtain $\tau_{\mathrm{cry}}=\mathrm{K}^{-1 / \mathrm{n}}$ and $\beta=\mathrm{n}$. Note that for each series of data, the value of $\tau_{\text {cry }}$ is systematically higher than the one of $t_{1 / 2}{ }^{D S}$. In fact the last corresponds to the condition $\varepsilon_{\mathrm{N}}^{\prime}\left(\mathrm{t}_{1 / 2}{ }^{\mathrm{DS}}\right)=0.5$ while the latter corresponds to $\varepsilon_{\mathrm{N}}^{\prime}\left(\tau_{\text {cry }}\right)=1-\mathrm{e}^{-1}=0.63$ and being $\varepsilon_{\mathrm{N}}^{\prime}(\mathrm{t})$ a monotonously growing function, the inequality $\varepsilon_{N}^{\prime}\left(\tau_{\text {cry }}\right)>\varepsilon_{N}^{\prime}\left(t_{1 / 2}{ }^{D S}\right)$ is always verified.

A regular behaviour is observed for the changes of bulk Avrami coefficients: $n \approx 3$ indicating a heterogeneous spherulitical growth, $\log \mathrm{K}$ scales with $-1 / \mathrm{T}$, see Figure 4. Lower values of the Avrami coefficients $(\mathrm{n} \rightarrow 2)$ were observed at the reduction of the thickness, as already reported ${ }^{16}$. This last trend implies that, considering the heterogeneous character of the process itself as being constant, the dimensionality of the crystallization process reduces by decreasing the thickness of the specimen. The experimental results will be discussed in the following paragraph together with several mechanisms proposed to explain the reduction of the crystallization rate (increase of $\tau_{\text {cry }}$ or decrease of the linear growth rate) and of the dimensionality (decrease of the Avrami exponent) in ultrathin polymer films.

\subsection{Slowing-down of the crystallization kinetics in ultrathin polymer films}

It was argued that the slowing-down of the crystallization kinetics can be imputed to a reduction of molecular mobility due to an increase of the glass transition of the system ${ }^{15}$, the presence of a layer showing a reduced mobility at the polymer/substrate interface ${ }^{17,18}$ and finally the reduction of the number of nuclei at a reduction of the thickness ${ }^{20-22}$.

As it was shown that a reduction of the crystallization kinetics is also observed in samples showing the same $^{18}$ or even a lower ${ }^{16}$ glass transition temperature compared to the one registered in bulk, the first mechanism described above seems inappropriate.

Instead, the presence of a layer with a different mobility at the very interface is supported by several experimental results obtained by different techniques ${ }^{7,17,39}$. A dynamic Monte Carlo simulation $^{40}$ confirmed that the crystalline content decreases in the proximity of an interacting interface (sticky walls) and that the crystallization

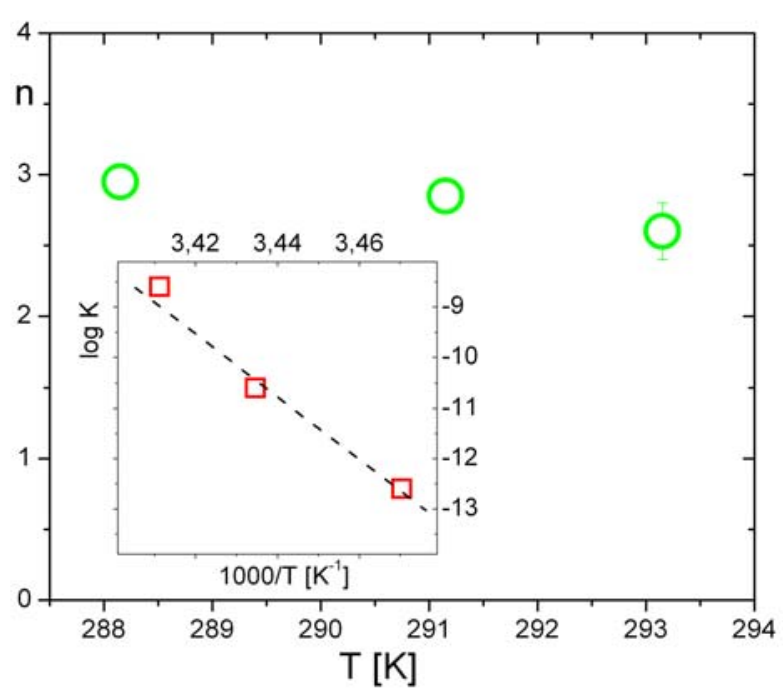

Figure 4. Temperature dependence of the Avrami parameters for bulk samples is inhibited by a highly interacting substrate. In contrast, weak interactions at the polymer/substrate interface (slippery walls) revealed a moderate enhancement of the crystallization kinetics at higher temperatures. Moreover it was found that the degree of polymer-substrate interactions is the essential parameter that governs the final geometry of the confined crystals. During an isothermal crystallization, both for slippery and for sticky walls, the polymer chains can rearrange themselves in lamellae, but in the case of sticky walls 
the molecules preferentially orientates along the direction normal to the substrate surface; while in presence of slippery walls, the crystals are orientated parallel to the surface of the substrate.

An intuitive explanation of the reduction of the value of $n$ in ultrathin films could be the effective reduction of the dimensionality of the crystal. Regardless the actual interaction with the substrate, in a confined geometry, spherulites cannot be formed due to both a spatial and dynamical confinement effects: spherical aggregates in fact do not fit the reduced dimensions of ultrathin films and likewise changes in the diffusion of material on the nanometre scale inhibit the crystals' growth.

Anyway, another mechanism could be responsible of the reduction of the Avrami parameter, a reduction not imputable to an effective lower dimensionality of the crystals' growth but to the reduced number of nuclei taking part to the conversion of the amorphous phase.

In fact, several models ${ }^{20-22}$ proposed corrections to the Avrami law, as this last is only strictly valid only in for the case of an infinite sample where crystallization can propagate to and from any position of the threedimensional space. Considering a reduced volume ${ }^{22}$, an ensemble of crystals will have nucleated outside the section of the considered space and thus should not be taken into account in the final dynamics.

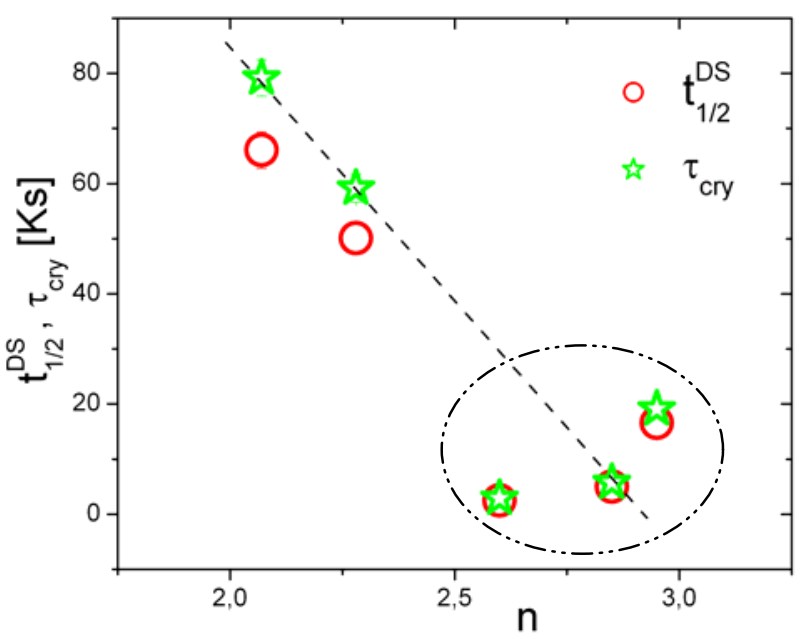

Figure 5. Correlation between reduction of the Avrami exponent and increase of the crystallization time, at a reduction of the sample thickness. Note that the values of $\tau_{\text {cry }}$ are systematically higher than $t_{1 / 2} \mathrm{DS}$, due to the different calculation procedure, see $\S 3.2$. The straight line is a guide for the eyes to follow the crystallization at $291 \mathrm{~K}$. The oval highlights the bulk data. Moreover an ensemble of the remaining crystals will have its own volume partially exceeding the considering space. As the two described ensembles do not contribute to the final dynamics, the crystallization rate in a reduced space ends up to be always lower than that in bulk. In particular, Schultz ${ }^{22}$ quantified the deviation from the bulk behaviour in a thin film geometry in terms of the thickness of the sample, $h$, and the linear growth factor of the crystal, G. The Avrami law gives results analyzable according to the classic crystallization theory only in the case of an infinite value of the ratio $h / G$. For polymers, being the values of $G$ typically of the order of $0.1-10$ $\mu \mathrm{m} / \mathrm{s}$, deviations from the bulk Avrami coefficient, $n(\infty)$, can be observed already for films thick a hundreds of nanometers ${ }^{16}$.

Experimentally, it is not possible to access the linear growth rate values of the samples investigated directly via dielectric spectroscopy. Even via imaging techniques, due to geometrical restrictions (the polymer film is capped between metallic layers) it would be definitely not possible to get an absolute value for the growth rate of the single crystals. A second complication is given by the fact that $\mathrm{G}$ is not constant in ultrathin films: the growth rate in fact, shows a non-monotone decrease ${ }^{14}$, by reducing the polymer thickness and it is 
thus not possible to extrapolate the bulk values of the investigated polymer. For relative variations of the thickness similar or larger than the connected relative variation of the crystal's growth factor $(d \ln G \leq d \operatorname{lnh})$, the ratio $\mathrm{h} / \mathrm{G}$ decreases upon reduction of the thickness. This condition is generally verified below $100 \mathrm{~nm}$ : thus the reduction of the film thickness corresponds to lower values of $h / G$, a lower value of $\eta(h)$ and consequently a larger deviation from the bulk behaviour $(h / G=\infty)$.

The experimental results of this work qualitatively comply with the model of Schultz: By reducing the thickness a deviation from the bulk behaviour is observed that manifests itself by an increase of the crystallization time together with a reduction of the corresponding Avrami exponent, see figure 5.

By comparing the effect of thickness reduction with the influence of temperature, see fig 5, we can conclude that the condition corresponding to a reduction of $\eta(h)$, i.e. increase of $\tau_{\text {cry }}$ coupled to a reduction of $n$, is not achieved by a simple reduction of the temperature.

\section{Conclusions}

A dielectric investigation of cold crystallization in ultrathin polymer films revealed an increase of the crystallization time together with a reduction of the apparent dimensionality of the process, expressed by a reduction of the Avrami exponent. The results have been rationalised considering different possible mechanisms leading to the dramatic changes observed in ultrathin samples. A possible explanation is the effective reduced dimensionality of the crystals' growth, the confinement in the thickness dimension impose a $2 \mathrm{D}$ reorganization of the polymer chains.

The experimental results qualitatively suggest also that the reduction of number of active nuclei in a reduced volume could be a cause of the slowing-down of crystallization kinetics in ultrathin polymer films.

\section{Acknowledgments}

S.N acknowledges financial support from the European Community's 'Marie-Curie Actions' under contract MRTN-CT-2004-504052 [POLYFILM].

\section{References}

(1) Alcoutlabi, M.; McKenna, G. B. Journal of Physics-Condensed Matter 2005, 17, R461R524.

(2) Chow, T. S. Journal of Physics-Condensed Matter 2002, 14, 1333-1339.

(3) Baschnagel, J.; Varnik, F. Journal of Physics-Condensed Matter 2005, 17, R851-R953.

(4) Meyer, H.; Baschnagel, J. European Physical Journal E 2003, 12, 147-151.

(5) Varnik, F.; Baschnagel, J.; Binder, K.; Mareschal, M. European Physical Journal E 2003, 12, 167-171.

(6) Keddie, J. L.; Jones, R. A. L.; Cory, R. A. Europhysics Letters 1994, 27, 59-64.

(7) Fukao, K.; Miyamoto, Y. Physical Review E 2000, 61, 1743-1754.

(8) Fryer, D. S.; Peters, R. D.; Kim, E. J.; Tomaszewski, J. E.; de Pablo, J. J.; Nealey, P. F.; White, C. C.; Wu, W. L. Macromolecules 2001, 34, 5627-5634. 
(9) Napolitano, S.; Prevosto, D.; Lucchesi, M.; Pingue, P.; D'Acunto, M.; Rolla, P. Langmuir, in press

(10) Despotopoulou, M. M.; Frank, C. W.; Miller, R. D.; Rabolt, J. F. Macromolecules 1996, 29, 5797-5804.

(11) Reiter, G.; Castelein, G.; Hoerner, P.; Riess, G.; Blumen, A.; Sommer, J. U. Physical Review Letters 1999, 83, 3844-3847.

(12) Reiter, G.; Sommer, J. U. Journal of Chemical Physics 2000, 112, 4376-4383.

(13) Reiter, G.; Vidal, L. European Physical Journal E 2003, 12, 497-505.

(14) Massa, M. V.; Dalnoki-Veress, K.; Forrest, J. A. European Physical Journal E 2003, 11, 191-198.

(15) Schonherr, H.; Frank, C. W. Macromolecules 2003, 36, 1199-1208.

(16) Zhang, Y.; Lu, Y. L.; Duan, Y. X.; Zhang, J. M.; Yan, S. K.; Shen, D. Y. Journal of Polymer Science Part B-Polymer Physics 2004, 42, 4440-4447.

(17) Capitan, M. J.; Rueda, D. R.; Ezquerra, T. A. Macromolecules 2004, 37, 5653-5659.

(18) Napolitano, S.; Wübbenhorst, M. Macromolecules 2006, 39, 5967-5970.

(19) Wurm, A.; Soliman, R.; Goossens, J. G. P.; Bras, W.; Schick, C. Journal of NonCrystalline Solids 2005, 351, 2773-2779.

(20) Escleine, J. M.; Monasse, B.; Wey, E.; Haudin, J. M. Colloid and Polymer Science 1984, 262, 366-373.

(21) Billon, N.; Escleine, J. M.; Haudin, J. M. Colloid and Polymer Science 1989, 267, 668-680.

(22) Schultz, J. M. Macromolecules 1996, 29, 3022-3024.

(23) Lupascu, V.; Huth, H.; Schick, C.; Wübbenhorst, M. Thermochimica Acta 2005, 432, 222228.

(24) Nogales, A.; Ezquerra, T. A.; Garcia, J. M.; Balta-Calleja, F. J. Journal of Polymer Science Part B-Polymer Physics 1999, 37, 37-49.

(25) Bergmann, A.; Owen, A. Polymer International 2004, 53, 863-868.

(26) Fukao, K.; Miyamoto, Y. Journal of Non-Crystalline Solids 1997, 212, 208-214.

(27) Fukao, K.; Miyamoto, Y. Journal of Non-Crystalline Solids 1998, 235, 534-538.

(28) Nogales, A.; Denchev, Z.; Sics, I.; Ezquerra, T. A. Macromolecules 2000, 33, 9367-9375.

(29) Nogales, A.; Ezquerra, T. A.; Denchev, Z.; Sics, I.; Calleja, F. J. B.; Hsiao, B. S. Journal of Chemical Physics 2001, 115, 3804-3813.

(30) Nogales, A.; Sics, I.; Ezquerra, T. A.; Denchev, Z.; Calleja, F. J. B.; Hsiao, B. S. Macromolecules 2003, 36, 4827-4832.

(31) Alvarez, C.; Sics, I.; Nogales, A.; Denchev, Z.; Funari, S. S.; Ezquerra, T. A. Polymer 2004, 45, 3953-3959.

(32) Mijovic, J.; Sy, J. W. Macromolecules 2002, 35, 6370-6376.

(33) Dionisio, M.; Viciosa, M. T.; Wang, Y. M.; Mano, J. F. Macromolecular Rapid Communications 2005, 26, 1423-1427.

(34) D'Amore, A.; Kenny, J. M.; Nicolais, L.; Tucci, V. Polymer Engineering and Science 1990, 30, 314-320.

(35) Massalska-Arodz, M.; Williams, G.; Thomas, D. K.; Jones, W. J.; Dabrowski, R. Journal of Physical Chemistry B 1999, 103, 4197-4205.

(36) Avrami, M. Journal of Chemical Physics 1940, 8, 212.

(37) Saad, G. R.; Mansour, A. A.; Hamed, A. H. Polymer 1997, 38, 4091-4096.

(38) Alie, J.; Menegotto, J.; Cardon, P.; Duplaa, H.; Caron, A.; Lacabanne, C.; Bauer, M. Journal of Pharmaceutical Sciences 2004, 93, 218-233.

(39) Forrest, J. A.; Mattsson, J. Physical Review E 2000, 61, R53-R56.

(40) Ma, Y.; Hu, W.; Reiter, G. Macromolecules 2006, 39, 5159-5164. 